Document downloaded from:

http://hdl.handle.net/10251/48226

This paper must be cited as:

Romaguera Bonilla, S.; Mujahid; Talat (2012). Fixed point results for generalized cyclic contraction mappings in partial metric spaces. Revista- Real Academia de Ciencias Exactas Fisicas Y Naturales Serie a Matematicas. 106(2):287-297. doi:10.1007/s13398011-0051-5.

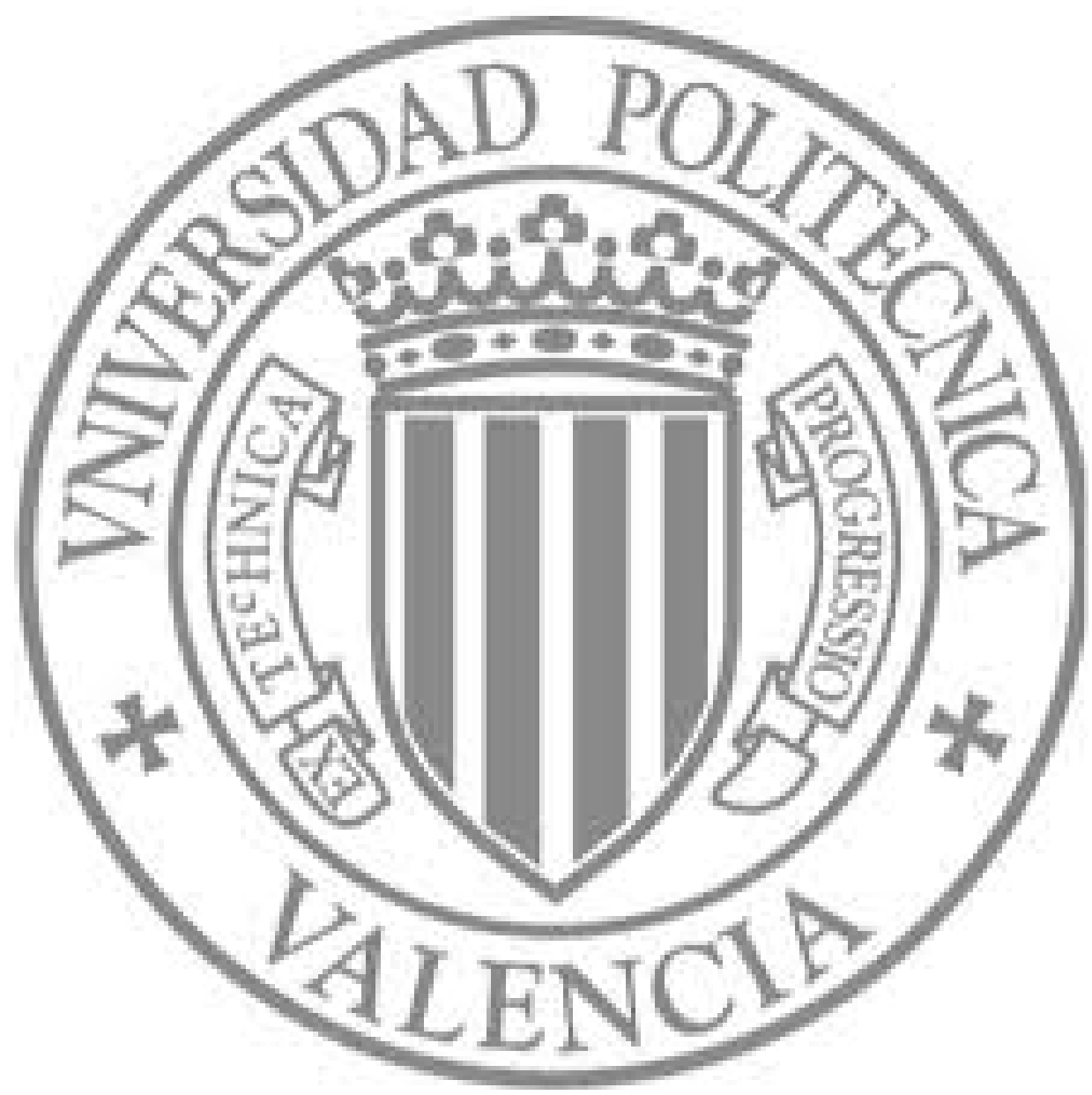

The final publication is available at

http://dx.doi.org/10.1007/s13398-011-0051-5

Copyright Springer Verlag (Germany) 


\title{
Fixed Point Results for Generalized Cyclic Contraction Mappings in Partial Metric Spaces
}

\author{
M. $\operatorname{Abbas}^{(1)}$, T. Nazir ${ }^{(2)}$, S. Romaguera ${ }^{(3)}$ \\ (1) The University of Birmingham, School of Mathematics, The Watson Building, \\ Edgbaston B15 2TT Birmingham, U. K and \\ Department of Mathematics, Lahore University of Management Sciences, 54792 \\ Lahore, Pakistan \\ ${ }^{(2)}$ Department of Mathematics, Lahore University of Management Sciences, 54792 \\ Lahore, Pakistan \\ (3) Instituto Universitario de Matemática Pura y Aplicada, Universitat Politècnica de \\ València, Camí de Vera s/n, 46022 Valencia, Spain
}

Abstract. Rus [Cyclic representations and fixed points, Ann. T. Popoviciu. Sem. Funct. Eq. Approx. Convexity 3 (2005), 171-178] introduced the concept of cyclic contraction mapping. Păcurar and Rus [Fixed point theory for cyclic $\phi$ - contractions, Nonlinear Anal. 72 (2010), 1181-1187] proved some fixed point results for cyclic $\phi$ - contraction mappings on a metric space. Karapinar [Fixed point theory for cyclic weak $\phi$ - contraction, Appl. Math. Lett. 24 (2011), $822-825$ ] obtained a unique fixed point of cyclic weak $\phi$ - contraction mappings and studied well-posedness problem for such mappings. On the other hand, Matthews [15] introduced the concept of a partial metric as a part of the study of denotational semantics of dataflow networks. He gave a modified version of the Banach contraction principle, more suitable in this context. In this paper, we initiate the study of fixed points of generalized cyclic contraction in the framework of partial metric spaces. We also present some examples to validate our results.

Keywords: Partial metric space, fixed point, cyclic contraction

\section{Introduction and preliminaries}

The study of fixed points of mappings satisfying cyclic contractive conditions has been at the center of vigorous research activity in the last years. In fact, in 2010, Păcurar and Rus [18] proved fixed point results for cyclic $\phi$-contractions. Karapinar [11] proved fixed point results for cyclic weak $\phi$-contraction. Recently, Karpagam and Agrawal [13] obtained best proximity point theorems for cyclic orbital Meir-Keeler contraction maps ( see also, [14], [16], [19] ).

On the other had, partial metric space is a generalized metric space in which each object does not necessarily have to have a zero distance from itself [15]. A motivation behind introducing the concept of a partial metric was to obtain appropriate mathematical models in the theory of computation $[10,15,22,23$,

\footnotetext{
${ }^{0}$ E-mail addresses: mujahid@lums.edu.pk (M. Abbas), talat@lums.edu.pk (T. Nazir), sromague@mat.upv.es (S. Romaguera).

The third author acknowledges the support of the Ministry of Science and Innovation of Spain, grant MTM2009-12872-C02-01.
} 
25, etc]. Altun and Simsek [3], Oltra and Valero [17] and Valero [24] established some further generalizations of the results in [15], and Romaguera [20] proved a Caristi type fixed point theorem on partial metric spaces. Further results in this direction were proved in $[4,1,5,8,7,12]$.

The aim of this paper is to study fixed point results for mappings satisfying generalized cyclic contractive conditions in the setup of partial metric spaces.

In the sequel the letters $\mathbb{R}, \mathbb{R}^{+}$and $N$ will denote the set of all real numbers, the set of all nonnegative real numbers and the set of all positive integer numbers, respectively.

Consistent with [3] and [15], the following definitions and results will be needed in the sequel.

Definition 1.1. Let $X$ be a nonempty set. A function $p: X \times X \rightarrow \mathbb{R}^{+}$is said to be a partial metric on $X$ if for any $x, y, z \in X$, the following conditions hold true:

$\left(\mathrm{P}_{1}\right) p(x, x)=p(y, y)=p(x, y)$ if and only if $x=y$;

$\left(\mathrm{P}_{2}\right) p(x, x) \leq p(x, y)$;

$\left(\mathrm{P}_{3}\right) p(x, y)=p(y, x)$

$\left(\mathrm{P}_{4}\right) p(x, z) \leq p(x, y)+p(y, z)-p(y, y)$.

The pair $(X, p)$ is then called a partial metric space.

If $p(x, y)=0$, then $\left(\mathrm{P}_{1}\right)$ and $\left(\mathrm{P}_{2}\right)$ imply that $x=y$. But the converse does not hold always.

A trivial example of a partial metric space is the pair $\left(\mathbb{R}^{+}, p\right)$, where $p$ : $\mathbb{R}^{+} \times \mathbb{R}^{+} \rightarrow \mathbb{R}^{+}$is defined as $p(x, y)=\max \{x, y\}$.

Example 1.2. [15] If $X=\{[a, b]: a, b \in \mathbb{R}, a \leq b\}$, then $p([a, b],[c, d])=$ $\max \{b, d\}-\min \{a, c\}$ defines a partial metric $p$ on $X$.

For some more examples of partial metric spaces, we refer to [3, 8, 20, 23].

Each partial metric $p$ on $X$ generates a $T_{0}$ topology $\tau_{p}$ on $X$ which has as a base the family open $p$-balls $\left\{B_{p}(x, \varepsilon): x \in X, \varepsilon>0\right\}$, where $B_{p}(x, \varepsilon)=\{y \in$ $X: p(x, y)<p(x, x)+\varepsilon\}$, for all $x \in X$ and $\varepsilon>0$.

Observe (see [15, p. 187]) that a sequence $\left\{x_{n}\right\}$ in a partial metric space $(X, p)$ converges to a point $x \in X$, with respect to $\tau_{p}$, if and only if $p(x, x)=$ $\lim _{n \rightarrow \infty} p\left(x, x_{n}\right)$.

If $p$ is a partial metric on $X$, then the function $p^{S}: X \times X \rightarrow \mathbb{R}^{+}$given by $p^{S}(x, y)=2 p(x, y)-p(x, x)-p(y, y)$, defines a metric on $X$.

Furthermore, a sequence $\left\{x_{n}\right\}$ converges in $\left(X, p^{S}\right)$ to a point $x \in X$ if and only if

$$
\lim _{n, m \rightarrow \infty} p\left(x_{n}, x_{m}\right)=\lim _{n \rightarrow \infty} p\left(x_{n}, x\right)=p(x, x) .
$$

Definition 1.3. [15] Let $(X, p)$ be a partial metric space. 
(a) A sequence $\left\{x_{n}\right\}$ in $X$ is said to be a Cauchy sequence if $\lim _{n, m \rightarrow \infty} p\left(x_{n}, x_{m}\right)$ exists and is finite.

(b) $(X, p)$ is said to be complete if every Cauchy sequence $\left\{x_{n}\right\}$ in $X$ converges with respect to $\tau_{p}$ to a point $x \in X$ such that $\lim _{n \rightarrow \infty} p\left(x, x_{n}\right)=p(x, x)$. In this case, we say that the partial metric $p$ is complete.

Lemma 1.4. $[3,15]$ Let $(X, p)$ be a partial metric space. Then:

(a) A sequence $\left\{x_{n}\right\}$ in $X$ is a Cauchy sequence in $(X, p)$ if and only if it is a Cauchy sequence in metric space $\left(X, p^{S}\right)$.

(b) A partial metric space $(X, p)$ is complete if and only if the metric space $\left(X, p^{S}\right)$ is complete.

Definition 1.5. [18] Let $X$ be a non-empty set and $f: X \rightarrow X$ an operator. By definition, $X=\cup_{i=1}^{m} X_{i}$ is a cyclic representation of $X$ with respect to $f$ if

(a) $X_{i}, i=1, \ldots, m$ are non-empty sets;

(b) $f\left(X_{1}\right) \subset X_{2}, \ldots, f\left(X_{m-1}\right) \subset X_{m}, f\left(X_{m}\right) \subset X_{1}$.

\section{$2 \quad$ Fixed point results}

In this section, we obtain several fixed point results for self maps satisfying certain generalized cyclic contractions defined on a complete partial metric space.

In the proof of our first result we shall use the following version of the celebrated Boyd and Wong fixed point theorem [6].

Theorem 2.1. $[4,21]$ Let $(X, p)$ be a complete partial metric space and let $f: X \rightarrow X$ be a mapping such that

$$
p(f x, f y) \leq \phi\left(\max \left\{p(x, y), p(f x, x), p(y, f y), \frac{p(x, f y)+p(y, f x)}{2}\right\}\right),
$$

for all $x, y \in X$, where $\phi:[0, \infty) \rightarrow[0, \infty)$ is a continuous function such that $\phi(t)<t$ for all $t>0$. Then $f$ has a unique fixed point.

Remark 2.2. Theorem 2.1 was initially established by Altun et al. in [4] (see also [2]) for the case that $\phi$ is a nondecreasing continuous function with $\phi(t)<t$ for all $t>0$. The version presented here was established by Romaguera in a more general form [21].

Theorem 2.3. Let $(X, p)$ be a complete partial metric space, $A_{1}, A_{2}, \ldots, A_{m}, m$ nonempty closed subsets of $\left(X, p^{S}\right)$ and $Y=\cup_{i=1}^{m} A_{i}$. Suppose that $f: Y \rightarrow Y$ is a mapping such that

(1) $\cup_{i=1}^{m} A_{i}$ is a cyclic representation of $Y$ with respect to $f$, and 
(2) there exists a continuous function $\phi:[0, \infty) \rightarrow[0, \infty)$ such that $\phi(t)<t$ for each $t>0$, satisfying

$$
p(f x, f y) \leq \phi(M(x, y)),
$$

for any $x \in A_{i}, y \in A_{i+1}, i=1,2, \ldots, m$, where $A_{m+1}=A_{1}$, and

$$
M(x, y)=\max \left\{p(x, y), p(f x, x), p(y, f y), \frac{p(x, f y)+p(y, f x)}{2}\right\},
$$

for any $x, y \in Y$. Then, $f$ has a unique fixed point $z \in Y$. Moreover $z \in \cap_{i=1}^{m} A_{i}$.

Proof. Let $x_{0}$ be an arbitrary point of $Y=\cup_{i=1}^{n} A_{i}$. Then there exists some $i_{0}$ such that $x_{0} \in A_{i_{0}}$. Now $f\left(A_{i_{0}}\right) \subseteq A_{i_{0}+1}$ implies that $f x_{0} \in A_{i_{0}+1}$. Thus there exists $x_{1}$ in $A_{i_{0}+1}$ such that $f x_{0}=x_{1}$. Similarly, $f x_{n}=x_{n+1}$, where $x_{n} \in A_{i_{n}}$. Hence for $n \geq 0$, there exists $i_{n} \in\{1,2, \ldots, m\}$ such that $x_{n} \in A_{i_{n}}$ and $x_{n+1} \in A_{i_{n+1}}$. In case, $x_{n_{0}}=x_{n_{0}+1}$ for some $n_{0}=0,1,2, \ldots$, then it is clear that $x_{n_{0}}$ is a fixed point of $f$. Now assume that $x_{n} \neq x_{n+1}$ for all $n$. Then, by (2.1), we have

$$
p\left(x_{n+1}, x_{n+2}\right)=p\left(f x_{n}, f x_{n+1}\right) \leq \phi\left(M\left(x_{n}, x_{n+1}\right)\right),
$$

where

$$
\begin{aligned}
M\left(x_{n}, x_{n+1}\right)= & \max \left\{p\left(x_{n}, x_{n+1}\right), p\left(f x_{n}, x_{n}\right), p\left(x_{n+1}, f x_{n+1}\right),\right. \\
& \left.\frac{1}{2}\left[p\left(x_{n}, f x_{n+1}\right)+p\left(x_{n+1}, f x_{n}\right)\right]\right\} \\
= & \max \left\{p\left(x_{n}, x_{n+1}\right), p\left(x_{n+1}, x_{n}\right), p\left(x_{n+1}, x_{n+2}\right),\right. \\
& \left.\frac{1}{2}\left[p\left(x_{n}, x_{n+2}\right)+p\left(x_{n+1}, x_{n+1}\right)\right]\right\} \\
\leq & \max \left\{p\left(x_{n}, x_{n+1}\right), p\left(x_{n+1}, x_{n+2}\right),\right. \\
& \left.\frac{1}{2}\left[p\left(x_{n}, x_{n+1}\right)+p\left(x_{n+1}, x_{n+2}\right)\right]\right\} \\
= & \max \left\{p\left(x_{n}, x_{n+1}\right), p\left(x_{n+1}, x_{n+2}\right)\right\} .
\end{aligned}
$$

So $M\left(x_{n}, x_{n+1}\right)=\max \left\{p\left(x_{n}, x_{n+1}\right), p\left(x_{n+1}, x_{n+2}\right)\right.$.

Suppose that $\max \left\{p\left(x_{k}, x_{k+1}\right), p\left(x_{k+1}, x_{k+2}\right)\right\}=p\left(x_{k+1}, x_{k+2}\right)$ for some $k \in$ $N$. Then $M\left(x_{k}, x_{k+1}\right)=p\left(x_{k+1}, x_{k+2}\right)$, so $p\left(x_{k+1}, x_{k+2}\right) \leq \phi\left(p\left(x_{k+1}, x_{k+2}\right)\right)<$ $p\left(x_{k+1}, x_{k+2}\right)$, a contradiction. Hence

$$
M\left(x_{n}, x_{n+1}\right)=p\left(x_{n}, x_{n+1}\right),
$$

and thus

$$
p\left(x_{n+1}, x_{n+2}\right) \leq \phi\left(p\left(x_{n}, x_{n+1}\right)\right)<p\left(x_{n}, x_{n+1}\right),
$$

for all $n \in N$. Therefore, the (decreasing) sequence of positive real numbers $\left\{p\left(x_{n}, x_{n+1}\right)\right\}$ converges to a $c \geq 0$. Then from (2.2) and the condition that $\phi$ is upper semicontinuous from the right, we obtain

$$
c=\lim _{n \rightarrow \infty} \phi\left(p\left(x_{n}, x_{n+1}\right)\right) \leq \phi(c) .
$$


Hence $c=0$ and thus $\lim _{n \rightarrow \infty} p\left(x_{n}, x_{n+1}\right)=0$.

Consequently, for any subsequence $\left\{x_{n_{k}}\right\}$ of $\left\{x_{n}\right\}$, one has $\lim _{k \rightarrow \infty} p\left(x_{n_{k}}, x_{n_{k}+1}\right)=$ 0 , and by condition (P4) in Definition 1.1, $\lim _{k \rightarrow \infty} p\left(x_{n_{k}}, x_{n_{k}+i}\right)=0$ for any $i \in N$.

The above fact will be used in the rest of the proof without explicit mention.

Now we shall prove that the sequence $\left\{x_{n}\right\}$ is a Cauchy sequence in $(Y, p)$.

In fact, since the sequence $\left\{x_{n}\right\}$ can be decomposed in $m$ subsequences $\left\{x_{n}^{(i)}\right\}$, $i=1, \ldots, m$, where $x_{n}^{(i)} \in A_{i}$, for each $n \in N$ t will be enough to prove that each subsequence $\left\{x _ { n } ^ { ( i ) } \longdiv { Q } = 1, \ldots, m\right.$, is a Caucrny sequence in $(Y, p)$.

In order to simpliry the argument we suppose, without loss of generality, that $x_{0} \in A_{m}$, so $x_{m} \in A_{m}$, and we shall prove that $\lim _{j, l \rightarrow \infty} p\left(x_{j}^{(m)}, x_{l}^{(m)}\right)=0$, i.e., $\lim _{j, l \rightarrow \infty} p\left(x_{m \cdot j}, x_{m \cdot l}\right)=0$.

Assume the contrary. Then there exists $\varepsilon>0$ and sequences $\left\{j_{k}\right\},\left\{l_{k}\right\}$, in $N$, with $l_{k}>j_{k} \geq k$, and such that $p\left(x_{m \cdot j_{k}}, x_{m \cdot l_{k}}\right) \geq \varepsilon$ and $p\left(x_{m \cdot j_{k}}, x_{m \cdot\left(l_{k}-1\right)}\right)<\varepsilon$ for all $k \in N$.

Now, for each $k \in N$ we have

$$
\begin{aligned}
\varepsilon & \leq p\left(x_{m \cdot j_{k}}, x_{m \cdot l_{k}}\right) \leq p\left(x_{m \cdot j_{k}}, x_{m \cdot\left(l_{k}-1\right)}\right)+p\left(x_{m \cdot\left(l_{k}-1\right)}, x_{m \cdot l_{k}}\right) \\
& <\varepsilon+p\left(x_{m \cdot\left(l_{k}-1\right)}, x_{m \cdot l_{k}}\right)
\end{aligned}
$$

so

$$
\lim _{k \rightarrow \infty} p\left(x_{m \cdot j_{k}}, x_{m \cdot l_{k}}\right)=\varepsilon
$$

Hence

$$
\lim _{k \rightarrow \infty} \phi\left(p\left(x_{m \cdot j_{k}}, x_{m \cdot l_{k}}\right)\right)=\phi(\varepsilon)<\varepsilon .
$$

Next we show that there is a subsequence of $\left\{M\left(x_{m \cdot j_{k}}, x_{m \cdot l_{k}-1}\right)\right\}$ that converges to some $\alpha \in[\varepsilon / 2, \varepsilon]$.

Indeed, for each $\delta \in(0, \varepsilon)$ there exists $k_{\delta} \in N$ such that for each $k \geq k_{\delta}$, $\delta$.

$p\left(x_{m \cdot j_{k}}, x_{m \cdot j_{k}+1}\right)<\delta, \quad p\left(x_{m \cdot l_{k}-m}, x_{m \cdot l_{k}-1}\right)<\delta \quad$ and $\quad p\left(x_{m \cdot l_{k}-1}, x_{m \cdot l_{k}}\right)<$

Moreover, since $p\left(x_{n_{k}}, x_{m_{k}-1}\right)<\varepsilon$, we deduce that

$$
\begin{aligned}
p\left(x_{m \cdot j_{k}}, x_{m \cdot l_{k}}\right) & \leq p\left(x_{m \cdot j_{k}}, x_{m \cdot l_{k}-m}\right)+p\left(x_{m \cdot l_{k}-m}, x_{m \cdot l_{k}-1}\right)+p\left(\left(x_{m \cdot l_{k}-1}, x_{m \cdot l_{k}}\right)\right. \\
& <\varepsilon+2 \delta,
\end{aligned}
$$

and

$$
\begin{aligned}
p\left(x_{m \cdot j_{k}+1}, x_{m \cdot l_{k}-1}\right) & \leq p\left(x_{m \cdot j_{k}+1}, x_{m \cdot j_{k}}\right)+p\left(x_{m \cdot j_{k}}, x_{m \cdot l_{k}-m}\right)+p\left(x_{m \cdot l_{k}-m}, x_{m \cdot l_{k}-1}\right) \\
& <2 \delta+\varepsilon,
\end{aligned}
$$


for all $k \geq k_{\delta}$. Therefore

$$
\begin{aligned}
\frac{\varepsilon}{2} \leq & \frac{p\left(x_{m \cdot j_{k}}, x_{m \cdot l_{k}}\right)}{2} \leq M\left(x_{m \cdot j_{k}}, x_{m \cdot l_{k}-1}\right) \\
= & \max \left\{p\left(x_{m \cdot j_{k}}, x_{m \cdot l_{k}-1}\right), p\left(x_{m \cdot j_{k}+1}, x_{m \cdot j_{k}}\right), p\left(x_{m \cdot l_{k}-1}, x_{m \cdot l_{k}}\right),\right. \\
& \left.\frac{p\left(x_{m \cdot j_{k}}, x_{m \cdot l_{k}}\right)+p\left(x_{m \cdot j_{k}+1}, x_{m \cdot l_{k}-1}\right)}{2}\right\} \\
< & \max \{\varepsilon+\delta, \delta, \delta, \varepsilon+2 \delta\}=\varepsilon+2 \delta,
\end{aligned}
$$

for all $k \geq k_{\delta}$. Hence, the sequence $\left\{M\left(x_{m \cdot j_{k}}, x_{m \cdot l_{k}-1}\right)\right\}$ has a subsequence $\left\{M\left(x_{m \cdot j_{k_{h}}}, x_{m \cdot l_{k_{h}}-1}\right)\right\}$ which converges to a real number $\alpha \in[\varepsilon / 2, \varepsilon]$, so

$$
\lim _{h \rightarrow \infty} \phi\left(M\left(x_{m \cdot j_{k_{h}}}, x_{m \cdot l_{k_{h}}-1}\right)\right)=\phi(\alpha)<\alpha \leq \varepsilon .
$$

Choose $\delta \in(\phi(\alpha), \alpha)$. Then, there is $h_{\delta} \in N$ such that $p\left(x_{m \cdot j_{k}}, x_{m \cdot j_{k_{h}}+1}\right)<$ $\delta-\phi(\alpha)$ for all $h \geq h_{\delta}$, and thus

$$
\begin{aligned}
p\left(x_{m \cdot j_{k_{h}}}, x_{m \cdot l_{k_{h}}}\right) & \leq p\left(x_{m \cdot j_{k_{h}}}, x_{m \cdot j_{k_{h}}+1}\right)+p\left(x_{m \cdot j_{k_{h}}+1}, x_{m \cdot l_{k_{h}}}\right) \\
& <\delta-\phi(\alpha)+\phi\left(M\left(x_{m \cdot j_{k_{h}}}, x_{m \cdot l_{k_{h}}-1}\right)\right)<\alpha
\end{aligned}
$$

for some $h \geq h_{\delta}$, which contradicts that $p\left(x_{m \cdot j_{k}}, x_{m \cdot l_{k}}\right) \geq \varepsilon$ for all $k \in N$.

We conclude that $\lim _{j, k \rightarrow \infty} p\left(x_{m \cdot j}, x_{m \cdot k}\right)=0$, i.e., $\left\{x_{n}^{(m)}\right\}$ is a Cauchy sequence in $(Y, p)$, and thus in the metric space $\left(Y, p^{S}\right)$ by Lemma 1.4. Similarly, we can prove that $\left\{x_{n}^{(i)}\right\}$ is a Cauchy sequence in $(Y, p)$, for $i=1, \ldots, m-1$, and consequently $\left\{x_{n}\right\}$ is a Cauchy sequence in $(Y, p)$, so in $\left(Y, p^{S}\right)$.

Since $Y$ is closed in $\left(X, p^{s}\right)$, then $\left(Y, p^{S}\right)$ is also complete. Thus, there exists $y_{0} \in Y$ such that $x_{n} \rightarrow y_{0}$ in $\left(Y, p^{S}\right)$; equivalently

$$
p\left(y_{0}, y_{0}\right)=\lim _{n \rightarrow \infty} p\left(y_{0}, x_{n}\right)=\lim _{n, m \rightarrow \infty} p\left(x_{n}, x_{m}\right)=0 .
$$

Notice that the iterative sequence $\left\{x_{n}\right\}$ has an infinite number of terms in $A_{i}$ for each $i=1, \ldots, m$. Hence, in each $A_{i}, i=1, \ldots, m$, we can construct a subsequence of $\left\{x_{n}\right\}$ that converges to $y$. Regarding that each $A_{i}, i=1, \ldots, m$, is closed, we conclude that $y_{0} \in \cap_{i=1}^{m} A_{i}$ and thus $\cap_{i=1}^{m} A_{i} \neq \emptyset$.

For simplicity, set $Z=\cap_{i=1}^{m} A_{i}$. Clearly, $Z$ is also closed and so $(Z, p)$ is complete. Consider the restriction of $f$ on $Z$, that is, $\left.f\right|_{Z}: Z \rightarrow Z$ (note that $\left.f\right|_{Z}$ is a self mapping of $Z$ by Definition 1.5 (b)). Then, $\left.f\right|_{Z}$ satisfies the assumptions of Theorem 2.1 and thus $\left.f\right|_{Z}$ has a unique fixed point in $Z$ (says $z)$.

Finally, suppose that there exists $u \in Y$ with $f u=u$ and $u \neq z$. Then

$$
p(u, z)=p(f u, f z) \leq \phi(M(u, z))
$$


where

$$
\begin{aligned}
M(u, z) & =\max \left\{p(u, z), p(u, f u), p(z, f z), \frac{1}{2}(p(u, f z)+p(f u, z))\right\} \\
& =\max \left\{p(u, z), p(u, u), p(z, z), \frac{1}{2}(p(u, z)+p(u, z))\right\} \\
& =p(u, z)
\end{aligned}
$$

so that

$$
p(u, z) \leq \phi(p(u, z)),
$$

which is a contradiction. Thus, $z$ is the unique fixed point of $f$.

Remark 2.4. Note that the proof of Theorem 2.3 shows that for any initial value $x \in Y$ we obtain an $y \in Z$ such that $f^{n} x \rightarrow y$ in $\left(Y, p^{S}\right)$. So, by Theorem 2.1, $\left.f\right|_{Z}$ has a unique fixed point $z$, which is, in fact, the unique fixed point of $f$. Hence $z$ is the unique fixed point for any initial starting point $x \in Y$.

If we take $\phi(t)=k t$ for $k \in[0,1)$ in Theorem 2.3, we have the following corollary.

Corollary 2.5. Let $(X, p)$ be a complete partial metric space, $A_{1}, A_{2}, \ldots, A_{m}$, $m$ nonempty closed subsets of $\left(X, p^{S}\right)$ and $Y=\cup_{i=1}^{m} A_{i}$. Suppose that $f: Y \rightarrow Y$ is a mapping such that

(1) $\cup_{i=1}^{m} A_{i}$ is a cyclic representation of $Y$ with respect to $f$, and

$$
p(f x, f y) \leq k M(x, y),
$$

for any $x \in A_{i}, y \in A_{i+1}, i=1,2, \ldots, m$, where $A_{m+1}=A_{1}$, and

$$
M(x, y)=\max \left\{p(x, y), p(f x, x), p(y, f y), \frac{p(x, f y)+p(y, f x)}{2}\right\},
$$

for any $x, y \in Y$. Then, $f$ has a unique fixed point $z \in Y$. Moreover $z \in \cap_{i=1}^{m} A_{i}$.

To prove our next result we need the following theorem due to Altun et al. $[4]$.

Theorem 2.6. [4] Let $(X, p)$ be a complete partial metric space and let $f: X \rightarrow X$ be a mapping such that

$$
p(f x, f y) \leq a_{1} p(x, y)+a_{2} p(f x, x)+a_{3} p(f y, y)+a_{4} p(f x, y)+a_{5} p(f y, x),
$$

for all $x, y \in X$, where $a_{i} \geq 0$ for $i=1,2, . ., 5$, such that if $a_{4} \geq a_{5}$, then $a_{1}+a_{2}+a_{3}+a_{4}+a_{5}<1$, and if $a_{4}<a_{5}$, then $a_{1}+a_{2}+a_{3}+a_{4}+2 a_{5}<1$. Then $f$ has a unique fixed point. 
Theorem 2.7. Let $(X, p)$ be a complete partial metric space, $A_{1}, A_{2}, \ldots, A_{m}, m$ non-empty closed subsets of $\left(X, p^{S}\right)$ and $Y=\cup_{i=1}^{m} A_{i}$. Suppose that $f: Y \rightarrow Y$ is a mapping such that $\cup_{i=1}^{m} A_{i}$, is a cyclic representation of $Y$ with respect to $f$, and

$$
p(f x, f y) \leq a_{1} p(x, y)+a_{2} p(f x, x)+a_{3} p(f y, y)+a_{4} p(f x, y)+a_{5} p(f y, x),
$$

for any $x \in A_{i}, y \in A_{i+1}, i=1,2, \ldots, m$, where $A_{m+1}=A_{1}$ and $a_{i} \geq 0$ for $i=1,2, . ., 5$, such that if $a_{4} \geq a_{5}$, then $a_{1}+a_{2}+a_{3}+a_{4}+a_{5}<1$, and if $a_{4}<a_{5}$, then $a_{1}+a_{2}+a_{3}+a_{4}+2 a_{5}<1$. Then, $f$ has a unique fixed point $z \in Y$. Moreover $z \in \cap_{i=1}^{m} A_{i}$.

Proof. Let $x_{0}$ be an arbitrary point of $Y=\cup_{i=1}^{n} A_{i}$. Following similar arguments to those given in the proof of Theorem 2.3, we construct a sequence $\left\{x_{n}\right\}$, where $f x_{n}=x_{n+1}, x_{n} \in A_{i_{n}}$ and $x_{n+1} \in A_{i_{n+1}}$, for some $i_{n} \in\{1,2, \ldots, m\}$, $n \in N$.

If $x_{n_{0}}=x_{n_{0}+1}$ for some $n_{0}=0,1,2, \ldots$, then it is clear that $x_{n_{0}}$ is a fixed point of $f$.

Now, by taking $p\left(x_{n}, x_{n+1}\right)>0$ for all $n \in N$, consider

$$
\begin{aligned}
p\left(x_{n+1}, x_{n+2}\right)= & p\left(f x_{n}, f x_{n+1}\right) \\
\leq & a_{1} p\left(x_{n}, x_{n+1}\right)+a_{2} p\left(f x_{n}, x_{n}\right)+a_{3} p\left(f x_{n+1}, x_{n+1}\right) \\
& +a_{4} p\left(f x_{n}, x_{n+1}\right)+a_{5} p\left(f x_{n+1}, x_{n}\right) \\
= & a_{1} p\left(x_{n}, x_{n+1}\right)+a_{2} p\left(x_{n+1}, x_{n}\right)+a_{3} p\left(x_{n+2}, x_{n+1}\right) \\
& +a_{4} p\left(x_{n+1}, x_{n+1}\right)+a_{5} p\left(x_{n+2}, x_{n}\right) \\
\leq & \left(a_{1}+a_{2}+a_{5}\right) p\left(x_{n}, x_{n+1}\right)+\left(a_{3}+a_{5}\right) p\left(x_{n+1}, x_{n+2}\right) \\
& +\left(a_{4}-a_{5}\right) p\left(x_{n+1}, x_{n+1}\right) .
\end{aligned}
$$

If $a_{4}<a_{5}$, then from $\left(a_{4}-a_{5}\right) p\left(x_{n+1}, x_{n+1}\right) \leq a_{4} p\left(x_{n}, x_{n+1}\right)$, it immediately follows

$$
p\left(x_{n+1}, x_{n+2}\right) \leq \lambda_{1} p\left(x_{n}, x_{n+1}\right),
$$

where $\lambda_{1}=\left(a_{1}+a_{2}+a_{4}+a_{5}\right) /\left(1-a_{3}-a_{5}\right)$.

If $a_{4} \geq a_{5}$, then from $\left(a_{4}-a_{5}\right) p\left(x_{n+1}, x_{n+1}\right) \leq\left(a_{4}-a_{5}\right) p\left(x_{n}, x_{n+1}\right)$, it immediately follows

$$
p\left(x_{n+1}, x_{n+2}\right) \leq \lambda_{2} p\left(x_{n}, x_{n+1}\right),
$$

where $\lambda_{2}=\left(a_{1}+a_{2}+a_{4}\right) /\left(1-a_{3}-a_{5}\right)$.

From both cases,

$$
p\left(x_{n+1}, x_{n+2}\right) \leq \lambda p\left(x_{n}, x_{n+1}\right),
$$

where $\lambda=\lambda_{1}$ if $a_{4}<a_{5}$, and $\lambda=\lambda_{2}$ if $a_{4} \geq a_{5}$. (Obviously, $0 \leq \lambda<1$ ).

Similarly it can be show that

$$
p\left(x_{n}, x_{n+1}\right) \leq \lambda p\left(x_{n-1}, x_{n-2}\right) .
$$


Thus for all $n \in N$, by repetition of the above process $n$ times, we have

$$
\begin{aligned}
p\left(x_{n}, x_{n+1}\right) & \leq \lambda p\left(x_{n-1}, x_{n}\right) \\
& \leq \ldots \leq \lambda^{n} p\left(x_{0}, x_{1}\right) .
\end{aligned}
$$

Therefore, for $m, n \in N$ with $m>n$, we have

$$
\begin{aligned}
p\left(x_{n}, x_{m}\right) & \leq p\left(x_{n}, x_{n+1}\right)+p\left(x_{n+1}, x_{n+2}\right)+\ldots+p\left(x_{m-1}, x_{m}\right) \\
& \leq\left(\lambda^{n}+\lambda^{n+1}+\ldots+\lambda^{m-1}\right) p\left(x_{0}, x_{1}\right)
\end{aligned}
$$

which implies that $\lim _{n, m \rightarrow \infty} p\left(x_{n}, x_{m}\right)=0$. So $\left\{x_{n}\right\}$ is a Cauchy sequence in $(Y, p)$. By Lemma 1.4, there exists $y_{0} \in Y$ such that $\lim _{n \rightarrow \infty} p^{S}\left(y_{0}, x_{n}\right)=0$, equivalently

$$
\lim _{n, m \rightarrow \infty} p\left(x_{n}, x_{m}\right)=\lim _{n \rightarrow \infty} p\left(y_{0}, x_{n}\right)=p\left(y_{0}, y_{0}\right)=0 .
$$

The same argument that we have in Theorem 2.3, shows that $y_{0} \in \cap_{i=1}^{m} A_{i}$ and thus $\cap_{i=1}^{m} A_{i} \neq \emptyset$.

For simplicity, set $Z=\cap_{i=1}^{m} A_{i}$. Clearly, $Z$ is also closed in $\left(X, p^{S}\right)$, so $(Z, p)$ is complete. Consider the restriction of $f$ on $Z$, that is, $\left.f\right|_{Z}: Z \rightarrow Z$ which obviously satisfies the assumptions of Theorem 2.6 and thus $\left.f\right|_{Z}$ has a unique fixed point in $Z$ (says $z$ ).

Finally, suppose that there exists $u \in Y$ with $f u=u$ and $u \neq z$. Then from (2.6), we have

$$
\begin{aligned}
p(u, z) & =p(f u, f z) \\
& \leq a_{1} p(u, z)+a_{2} p(f u, u)+a_{3} p(f z, z)+a_{4} p(f u, z)+a_{5} p(f z, u) \\
& =a_{1} p(u, z)+a_{2} p(u, u)+a_{3} p(z, z)+a_{4} p(u, z)+a_{5} p(u, z) \\
& \leq\left(a_{1}+a_{2}+a_{3}+a_{4}+a_{5}\right) p(u, z),
\end{aligned}
$$

which is a contradiction. Thus, $z$ is the unique fixed point of $f$.

Note that, as in Theorem 2.3 (compare Remark 2.4), $z$ is the unique fixed point for any initial starting point $x \in Y$.

Corollary 2.8. Let $(X, p)$ be a complete partial metric space, $A_{1}, A_{2}, \ldots, A_{m}$, $m$ non-empty closed subsets of $\left(X, p^{S}\right)$ and $Y=\cup_{i=1}^{m} A_{i}$. Suppose that $f: Y \rightarrow$ $Y$ is a mapping such that $\cup_{i=1}^{m} A_{i}$, is a cyclic representation of $Y$ with respect to $f$, and

$$
p(f x, f y) \leq \alpha p(x, y)+\beta[p(f x, x)+p(f y, y)]+\gamma[p(f x, y)+p(f y, x)]
$$

for any $x \in A_{i}, y \in A_{i+1}, i=1,2, \ldots, m$, where $A_{m+1}=A_{1}$ and $\alpha, \beta, \gamma \geq 0$ and $\alpha+2 \beta+2 \gamma<1$. Then, $f$ has a unique fixed point $z \in Y$. Moreover $z \in \cap_{i=1}^{m} A_{i}$.

We finish the paper with two examples that illustrate and validate Theorems 2.3 and 2.7 , respectively. 
Example 2.9. Let $(X, p)$ be the partial metric space of Example 1.2.

It is well known that $(X, p)$ is complete. In fact, observe that for each $[a, b],[c, d] \in X$ one has

$$
p^{S}([a, b],[c, d])=2(\max \{b, d\}-\min \{a, c\})-(b-a)-(d-c),
$$

so, for a Cauchy sequence $\left\{\left[a_{n}, b_{n}\right]\right\}$ in $\left(X, p^{s}\right)$, it easily follows that both $\left\{a_{n}\right\}$ and $\left\{b_{n}\right\}$ are Cauchy sequences in $\mathbb{R}$ for the Euclidean metric. Then $\lim _{n \rightarrow \infty} p^{S}\left(\left[a_{n}, b_{n}\right],[a, b]\right)=0$, where $a=\lim _{n \rightarrow \infty} a_{n}$ and $b=\lim _{n \rightarrow \infty} b_{n}$ in $\mathbb{R}$.

Suppose $A_{1}=\left\{a_{n}: n \in N\right\} \cup\{\{1\}\}$, where $a_{n}=\left[1-2^{-n}, 1\right]$ for all $n \in N$, $A_{2}=\left\{b_{n}: n \in N\right\} \cup\{\{1\}\}$, where $b_{n}=\left[1,1+2^{n}\right]$ for all $n \in N, A_{3}=A_{1}$, and $Y=A_{1} \cup A_{2}$.

Now define $f: Y \rightarrow Y$ by $f\{1\}=f b_{n}=\{1\}$ and $f a_{n}=b_{n}$ for all $n \in N$.

Since $p^{S}\left(\{1\}, a_{n}\right)=3 \cdot 2^{-n}$, we deduce that $\lim _{n \rightarrow \infty} p^{S}\left(\{1\}, a_{n}\right)=0$, and thus $A_{1}$ is closed in $\left(X, p^{S}\right)$. On the other hand, $p^{S}\left(\{1\}, b_{n}\right)=2^{n}$ and $p^{S}\left(b_{n}, b_{k}\right)=$ $\left|2^{n}-2^{k}\right|$ for all $n, k \in N$, so $A_{2}$ is closed in $\left(X, p^{S}\right)$. Moreover $f\left(A_{i}\right) \subset A_{i+1}$ for $i=1,2$, so that $A_{1} \cup A_{2}$ is a cyclic representation of $Y$ with respect to $f$.

Define $\Phi:[0, \infty) \rightarrow[0, \infty)$ by $\Phi(t)=4 t / 5$ whenever $t \in[0,5 / 2)$, and

$$
\Phi(t)=\frac{2^{2 n+1}}{2^{2 n+1}-1} t-\frac{3 \cdot 2^{n}}{2^{2 n+1}-1},
$$

whenever $t \in\left[2^{n}+2^{-n}, 2^{n+1}+2^{-(n+1)}\right), n \in N$.

An easy computation shows that $\Phi$ is continuous on $[0, \infty)$ and $\Phi(t)<t$ for all $t>0$. (In fact, the graph of the restriction of $\Phi$ to $\left[2^{n}+2^{-n}, 2^{n+1}+2^{-(n+1)}\right]$ is the straight line segment with origin at $\left(2^{n}+2^{-n}, 2^{n}\right)$ and end at $\left(2^{n+1}+\right.$ $\left.2^{-(n+1)}, 2^{n+1}\right)$.)

Next we show that contraction (2.1) is verified. Indeed, we have

$$
\begin{aligned}
p\left(f a_{n}, f\{1\}\right) & =p\left(b_{n}, 1\right)=2^{n}=\Phi\left(2^{n}+2^{-n}\right) \\
& =\Phi\left(p\left(b_{n}, a_{n}\right)\right)=\Phi\left(p\left(f a_{n}, a_{n}\right)\right)=\Phi\left(M\left(a_{n},\{1\}\right)\right),
\end{aligned}
$$

for all $n \in N$, and thus

$$
p\left(f a_{n}, f b_{k}\right)=p\left(b_{n},\{1\}\right)=2^{n}=\Phi\left(p\left(f a_{n}, a_{n}\right)\right) \leq \Phi\left(M\left(a_{n}, b_{k}\right)\right),
$$

for all $n, k \in N$.

Moreover $p\left(f b_{n}, f\{1\}\right)=0$ for all $n \in N$, so the conditions of Theorem 2.3 are satisfied.

Observe that $\{1\}$ is the unique fixed point of $X$. However $f$ is not continuous at $\{1\}$ for $\tau_{p^{S}}$ because $\lim _{n \rightarrow \infty} p^{S}\left(a_{n},\{1\}\right)=0$ but $p^{S}\left(f a_{n}, f\{1\}\right)=2^{n}$ for all $n \in N$. In fact, $f$ is not continuous at $\{1\}$ neither for $\tau_{p}$ because $\lim _{n \rightarrow \infty} p\left(a_{n},\{1\}\right)=\lim _{n \rightarrow \infty} 2^{-n}=0=p(\{1\},\{1\})$ but $p\left(f a_{n}, f\{1\}\right)=2^{n}$ for all $n \in N$.

Example 2.10. Let $X=[0,2]$. Let $p: X \times X \rightarrow \mathbb{R}^{+}$defined by $p(x, y)=$ $|x-y|$ if $x, y \in[0,1)$, and $p(x, y)=\max \{x, y\}$ otherwise. It is easily seen that $(X, p)$ is a complete partial metric space. 
Suppose $A_{1}=[0,1 / 2], A_{2}=[1 / 2,1], A_{3}=A_{1}$, and $Y=A_{1} \cup A_{2}=[0,1]$.

Now define $f: Y \rightarrow Y$ by $f x=1 / 2$ if $x \in[0,1)$ and $f 1=0$.

Clearly, $A_{1}$ and $A_{2}$ are closed subsets of $\left(X, p^{S}\right)$. Moreover $f\left(A_{i}\right) \subset A_{i+1}$ for $i=1,2$, so that $A_{1} \cup A_{2}$ is a cyclic representation of $Y$ with respect to $f$.

Next we show that contraction (2.6) is verified for $a_{i}=1 / 6$ for $i=1, \ldots, 5$. We shall distinguish the following cases.

(1) If $x \in A_{1}, y \in A_{2}$, then for $x \in[0,1 / 2]$ and $y \in[1 / 2,1)$, we deduce

$$
p(f x, f y)=p\left(\frac{1}{2}, \frac{1}{2}\right)=0,
$$

and when $x \in[0,1 / 2]$ and $y=1$, we deduce

$$
\begin{aligned}
p(f x, f y) & =p\left(\frac{1}{2}, 0\right)=\frac{1}{2}<\frac{1}{6}+\frac{1}{6}\left(\frac{1}{2}-x\right)+\frac{1}{6}+\frac{1}{6}+\frac{x}{6} \\
& =a_{1} p(x, y)+a_{2} p(f x, x)+a_{3} p(f y, y)+a_{4} p(f x, y)+a_{5} p(f y, x) .
\end{aligned}
$$

(2) If $x \in A_{2}, y \in A_{1}$, then for $x \in[1 / 2,1), y \in[0,1 / 2]$, we deduce

$$
p(f x, f y)=p\left(\frac{1}{2}, \frac{1}{2}\right)=0,
$$

and when $x=1$ and $y \in[0,1 / 2]$, we deduce

$$
\begin{aligned}
p(f x, f y) & =p\left(0, \frac{1}{2}\right)=\frac{1}{2}<\frac{1}{6}+\frac{1}{6}+\frac{1}{6}\left(\frac{1}{2}-y\right)+\frac{y}{6}+\frac{1}{6} \\
& =a_{1} p(x, y)+a_{2} p(f x, x)+a_{3} p(f y, y)+a_{4} p(f x, y)+a_{5} p(f y, x) .
\end{aligned}
$$

Hence $f$ verifies contaction (2.6), so the conditions of Theorem 2.7 are satisfied. Moreover, $1 / 2$ is the unique fixed point of $f$, and $f$ is not continuous for $\tau_{p^{S}}$ at 1.

\section{References}

[1] I. Altun and A. Erduran, Fixed point theorems for monotone mappings on partial metric spaces, Fixed Point Theory Appl. 2011 (2011), article ID 508730,10 pages.

[2] I. Altun and K. Sadarangani, Corrigendum to "Generalized contractions on partial metric spaces" [Topology Appl. 157 (2010), 2778-2785], Topology Appl. 158 (2011), 1738-1740.

[3] I. Altun and H. Simsek, Some fixed point theorems on dualistic partial metric spaces, J. Adv. Math. Stud. 1 (2008), 1-8.

[4] I. Altun, F. Sola and H. Simsek, Generalized contractions on partial metric spaces, Topology Appl. 157 (2010), 2778-2785. 
[5] H. Aydi, Some fixed point results in ordered partial metric spaces, arxiv: 1103.3680v1 [math.GN], (2011), 1-8.

[6] D. W. Boyd and J. S. W. Wong, On nonlinear contractions, Proc. Amer. Math. Soc. 20 (1969), 458-464.

[7] M. Bukatin, R. Kopperman, S. Matthews and H. Pajoohesh, Partial metric spaces, Amer. Math. Monthly 116 (2009), 708-718.

[8] M. A. Bukatin and S. Yu. Shorina, Partial metrics and co-continuous valuations, in: M. Nivat, et al. (Eds.), Foundations of Software Science and Computation Structure, in: Lecture Notes in Computer Science, vol. 1378, Springer, 1998, pp. 125-139.

[9] M. Derafshpour, S. Rezapour and N. Shahzad, On the existence of best proximity points of cyclic contractions, Adv. Dynam. Sys. Appl. 6 (2011), $33-40$.

[10] R. Heckmann, Approximation of metric spaces by partial metric spaces, Appl. Cat. Struct. 7 (1999), 71-83.

[11] E. Karapinar, Fixed point theory for cyclic weak $\phi$ - contraction, App. Math. Lett. 24 (2011), 822-825.

[12] E. Karapinar and I. M. Erhan, Fixed point theorems for operators on partial metric spaces, Appl. Math. Lett. 24 (2011), 1894-1899.

[13] S. Karpagam and S. Agrawal, Best proximity point theorems for cyclic orbital Meir-Keeler contraction maps, Nonlinear Anal. 74 (2011), 10401046 .

[14] G. S. R. Kosuru and P. Veeramani, Cyclic contractions and best proximity pair theorems, arXiv:1012.1434v2 [math.FA] 29 May 2011, 14 pages.

[15] S. G. Matthews, Partial metric topology, in: Proc. 8th Summer Conference on General Topology and Applications, Ann. New York Acad. Sci. 728 (1994), 183-197.

[16] K. Neammanee and A. Kaewkhao, Fixed points and best proximity points for multi-valued mapping satisfying cyclical condition, Int. J. Math. Sci. Appl. 1 (2011), 9 pages.

[17] S. Oltra and O. Valero, Banach's fixed theorem for partial metric spaces, Rend. Istit. Mat. Univ. Trieste 36 (2004), 17-26.

[18] M. Păcurar and I. A. Rus, Fixed point theory for cyclic $\phi$-contractions, Nonlinear Anal. 72 (2010), 1181-1187.

[19] M. A Petric, Best proximity point theorems for weak cyclic Kannan contractions, Filomat 25 (2011), 145-154. 
[20] S. Romaguera, A Kirk type characterization of completeness for partial metric spaces, Fixed Point Theory Appl., 2010 (2010), article ID 493298, 6 pages.

[21] S. Romaguera, Fixed point theorems for generalized contractions on partial metric spaces, submitted.

[22] S. Romaguera and O. Valero, A quantitative computational model for complete partial metric spaces via formal balls, Math. Struct. Comput. Sci. 19 (2009), 541-563.

[23] M. P. Schellekens, The correspondence between partial metrics and semivaluations, Theoret. Comput. Sci. 315 (2004), 135-149.

[24] O. Valero, On Banach fixed point theorems for partial metric spaces, Appl. Gen. Top. 6 (2005), 229-240.

[25] P. Waszkiewicz, Quantitative continuous domains, Appl. Cat. Struct. 11 (2003), 41-67. 